\title{
An Alternative Approach to the Gaussian Noise Model and its System Implications
}

\author{
Paolo Serena, Member, IEEE, and Alberto Bononi, Senior Member, IEEE
}

\begin{abstract}
This paper presents an alternative derivation of the Gaussian noise (GN) model of the nonlinear interference (NLI) in strongly dispersive optical systems. The basic idea is to exploit an enhanced regular perturbation expansion of the NLI, which highlights several interesting features of the GN model. Using the framework, we derive a fast algorithm to evaluate the received NLI power spectral density (PSD) for any input PSD. In the paper we also provide an asymptotic expression of the NLI PSD which further speeds up the computation without losing significant accuracy. Moreover, we show how the asymptotic expression can be used to optimize system performance. For instance, we are able to prove why a flat spectrum is best to minimize the NLI variance when the input is constrained to a fixed bandwidth and power. Finally, we also provide a generalization of the NLI GN model to arbitrarily correlated $\mathrm{X}$ and $\mathrm{Y}$ polarizations.
\end{abstract}

Index Terms-Gaussian Noise (GN) model, perturbation methods.

\section{INTRODUCTION}

D ISPERSION-Uncompensated (DU) coherent systems are becoming the new paradigm for high rate long-haul optical transmission systems and networks. The distinctive feature of these highly dispersive systems is a Gaussian distribution of the received sampled signal [1], [2], a consequence of the addition of very many comparable-size nonlinear field interferers from symbol times even far away from the symbol of interest. The Gaussian nature of both the nonlinear interference (NLI) and the amplified spontaneous emission noise fields brings the theory of these nonlinear optical coherent communication systems back to the standard additive Gaussian noise (GN) model of most nonoptical telecommunications literature, and allows elementary system optimization rules based on the electrical signal to noise ratio [3]-[5].

Several analytical models for performance evaluation and design of such systems have been proposed [6]-[10], which all share the same underlying basic assumption that nonlinearity is a small perturbation of the nonlinear Schrödinger equation (NLSE) of propagation. Among these, the GN model developed in [8], [10] is particularly attractive since it is able to provide a

Manuscript received July 11, 2013; revised September 24, 2013; accepted September 28, 2013. Date of publication October 3, 2013; date of current version October 23, 2013. This work was supported in part by a grant from Alcatel Lucent, Bell Labs, Centre de Villarceaux, Paris (France).

The authors are with the Department of Information Engineering, Università degli Studi di Parma, v.le G. Usberti 181/a, 43124, Parma, Italy (e-mail: paolo.serena@unipr.it; alberto.bononi@unipr.it).

Color versions of one or more of the figures in this paper are available online at http://ieeexplore.ieee.org.

Digital Object Identifier 10.1109/JLT.2013.2284499 simple formula for the NLI power spectral density (PSD), the so called GN reference formula (GNRF). The GN model is based on two fundamental assumptions:

1) the NLI field can be approximated by the first-order regular perturbation (RP) method;

2) the signal at the input of the nonlinear optical link is a stationary circular complex Gaussian process.

We showed in [11] that the errors in performance estimation induced by these two assumptions partially compensate each other, yielding good predictions of the GNRF.

The detailed proof of the GNRF with uncorrelated $\mathrm{X}$ and $\mathrm{Y}$ polarizations was provided by Johannisson et al. [12] and by Poggiolini et al. [13]. In [14] we proposed an alternative proof working in the frequency domain. All these proofs start from the main integral equation describing the RP NLI field [6], [15] as a sum of cubic beating terms weighted by the kernel of the integral equation.

The formal elegance and simplicity of the GNRF is remarkable, but despite approximate explicit solutions exist in special cases [10], the numerical evaluation of the NLI PSD using the GNRF for a generic input PSD is still a hard task, calling for a complexity of the order of $O\left(M^{3}\right)$, with $M$ the number of discrete frequencies of the input PSD.

In this paper we provide a new look at the GN model which does not stem form the kernel-based integral equation. We indeed exploit the enhanced-RP (eRP) method which allows to avoid extra secular terms in the RP expansion. Thanks to its formal simplicity, the idea can be used to write a very fast RP-based algorithm with complexity $O(M \log M)$ for the accurate solution of the NLI PSD, whatever the input PSD. The algorithm can be applied to periodic dispersion managed (DM) optical links, whereof DU are a special case. Moreover, we show that the proposed algorithm also provides additional insight into the main features of the GN model, e.g., a simple proof that the optimal input PSD minimizing the NLI variance over a fixed bandwidth is flat in frequency. By means of asymptotic expansion theory, we also show interesting approximations of the GNRF that can further speed up the numerical computation of the NLI PSD. We finally show in an Appendix how using the new approach the model can be extended to the dual-polarization case with possibly correlated $\mathrm{X}$ and $\mathrm{Y}$ polarizations.

The paper is organized as follows. In Section II we review the basic RP and eRP theory, showing some important implications in DU systems and different numerical algorithms for their solution. In Section III we provide the new proof of the GNRF using the eRP method. In Section IV we use asymptotic expansion theory to provide approximations of the GNRF in the new eRP formulation. We show that the proposed expansion 
can drastically speed up the numerical solution of the NLI PSD without losing in accuracy. Using the asymptotic expansion of the NLI PSD we are also able to infer the best shape of the transmitted PSD constrained to a fixed bandwidth and power.

\section{RP MODELS}

A considerable amount of literature has been published on the perturbative solution of the NLSE [16]-[21]. The general approach based on Volterra series expansion [20] has been shown to coincide with the RP solution of the NLSE [18]. Generally speaking, the electric field ${ }^{1} \tilde{A}(L, \omega)$ after distance $L$ and at angular frequency $\omega$ under the RP first order approximation (RP1) can be written [22] as $\tilde{A}(L, \omega) \simeq \tilde{A}_{0}(L, \omega)+\gamma \tilde{A}_{1}(L, \omega)$, with $\gamma$ the nonlinear coefficient of the optical fiber. $\tilde{A}_{0}(L, \omega)$ is the unperturbed solution of the NLSE, thus affected by linear effects only. $\tilde{A}_{1}(L, \omega)$ accounts for a first-order perturbation of the Kerr effect, and may be interpreted as a sum of first-order four wave mixing (FWM) fields among signal spectral lines [10], [23]. The general RP1 theory requires $\gamma$ to be a "small parameter", such that a Taylor series in $\gamma$ can be safely truncated to first order. However, $\gamma$ is generally a function of the propagation coordinate, hence it cannot be treated as just a parameter. A simple workaround is to artificially multiply the nonlinear term of the NLSE by a dummy parameter, say $\epsilon$, and then to search for a RP expansion in terms of $\epsilon$ [22]. Finally, setting $\epsilon=1$ yields the desired RP approximation. In the following we will use this point of view.

The basic RP1 solution generally fails in approximating the exact solution if the reference system under which the Taylor expansion is performed is not appropriately chosen. Since the Kerr effect manifests first of all as a self phase modulation (SPM), in [18] it was shown that deriving the RP1 in a reference system rotated by a proper time-constant phase is more accurate than in the original reference system at the transmitter. The corresponding RP method, called the eRP, was shown [18, (31)] to be identical to the RP method by substituting the nonlinear term $|A|^{2} A$ of the normalized NLSE with $\left(|A|^{2}-\hat{P}\right) A$. In single-channel $10 \mathrm{~Gb} / \mathrm{s}$ on-off keying (OOK) transmissions over DM systems the power $\hat{P}$ was chosen equal to the transmitted peak power [18]. The rationale was that in the small dispersion regime an OOK constellation remains almost unchanged in such an SPM-rotated reference system, thus justifying an RP expansion. However, since a DU system works in the largedispersion regime, we investigated the SPM-induced phase rotation by numerical simulation. We estimated the nonlinear phase experienced by a quadrature phase shift keying (QPSK) signal at pulse centers, after scalar propagation in a DU system with different values of dispersion. The link was DU, $20 \times 100$ $\mathrm{km}$ long, with total dispersion compensation at the end of the link. Fibers, modeled by the split step Fourier method (SSFM), had attenuation $0.2 \mathrm{~dB} / \mathrm{km}$, variable dispersion, nonlinear index $\gamma=1.31 / \mathrm{mW} / \mathrm{km}$. The transmitted signal had average power of $-3 \mathrm{dBm}$ and symbol rate $R=28$ Gbaud. The elementary pulses

\footnotetext{
${ }^{1}$ The Fourier transform is defined here as $\tilde{A}(L, \omega)=\mathcal{F}\{A(L, t)\}=$ $\int_{-\infty}^{\infty} A(L, t) e^{-j \omega t} d t$.
}
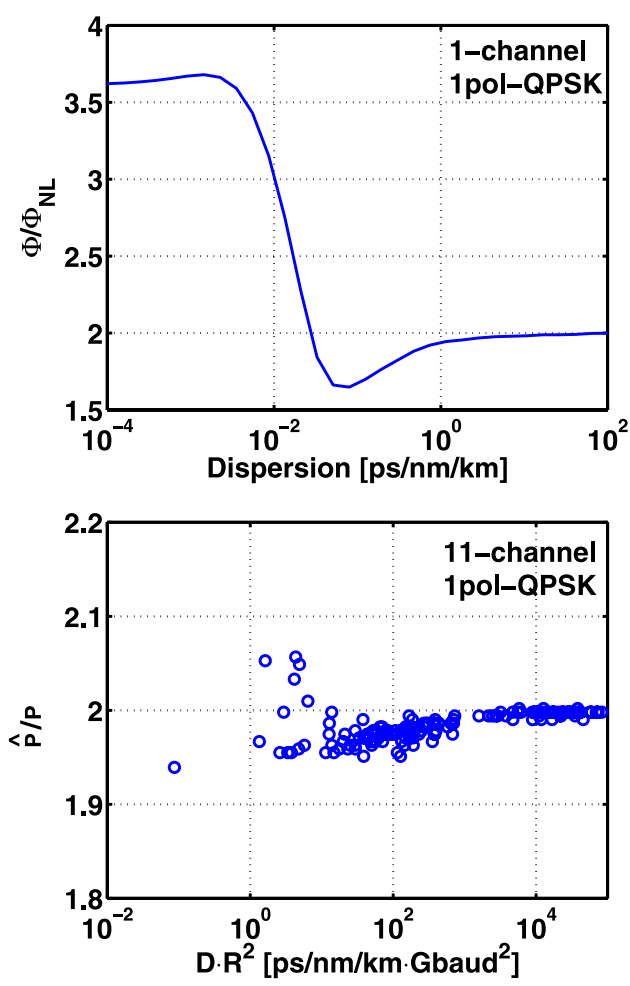

Fig. 1. Top: Ratio between the SSFM-estimated phase $\Phi$ induced by the Kerr effect at the pulse centers and the theoretical average phase $\Phi_{\mathrm{NL}}$ versus fiber dispersion D. $20 \times 100 \mathrm{~km}$ DU link. Single channel, QPSK at $R=28$ Gbaud. Bottom: best $\hat{P} / P$ minimizing the standard deviation of the error between eRP and SSFM. $N \times 100 \mathrm{~km} 11$ channel QPSK transmission with random $D$, $N$ and $R$. For reference, a $D=17 \mathrm{ps} / \mathrm{nm} / \mathrm{km}$ link working at 28 Gbaud has $D \cdot R^{2}=13328\left[\mathrm{ps} / \mathrm{nm} / \mathrm{km} \cdot \mathrm{Gbaud}^{2}\right]$.

were Gaussian shaped by $\exp \left(-t^{2} /\left(2 T_{0}^{2}\right)\right)$ with $T_{0}=1 /(6 R)$, which well emulated a $33 \%$ duty cycle transmission. We used Gaussian pulses since a straightforward application of the theory in [16] allowed us to build an analytical model (not reported here) that cross-validated the single-channel result in Fig. 1, to be described next.

For the above periodic system, the nonlinear phase induced by SPM over a constant signal of power $P$ is [24]

$$
\Phi_{\mathrm{NL}}(P)=\gamma P L_{\mathrm{eff}} N .
$$

$L_{\text {eff }}$ being the fiber effective length and $N$ the number of spans. The ratio between the nonlinear phase estimated at pulse centers, called $\Phi$, and $\Phi_{\mathrm{NL}}(P)$, with $P$ the average power of the train of pulses, is plotted in Fig. 1 (top) versus fiber dispersion at $P=-3 \mathrm{dBm}$. It is worth noting that the ratio saturates to 2 at practical dispersions greater than $1 \mathrm{ps} / \mathrm{nm} / \mathrm{km}$, so that in the large dispersion regime the nonlinear phase is 2 times the nonlinear phase induced by the average power, as previously observed in [25], [13]. At small dispersions the factor saturates instead to 3.65 , which is the peak-to-average power ratio of the transmitted signal, confirming that in the small dispersion regime of typical DM systems the peak nonlinear phase must be considered, as in [18]. According to the eRP idea, choosing $\hat{P}=\Phi /\left(\gamma L_{\text {eff }} N\right)$ such that the new reference system tracks the nonlinear induced phase rotation improves the accuracy of the RP perturbation. 
The dependence of $\hat{P}$ on dispersion observed in singlechannel transmission does not appear in practical wavelength division multiplexed (WDM) DU systems. To support this claim, we simulated a scalar 11 channel DU system, QPSK modulated with non-return to zero pulses, $50 \mathrm{GHz}$ channel spacing. We selected the following parameters according to a uniform random distribution: dispersion from 0.1 to $100 \mathrm{ps} / \mathrm{nm} / \mathrm{km}$; number of spans from 4 to 30 ; symbol rate from 10 to 32 Gbaud. 100 different seeds were tested and we measured the corresponding best value of power $\hat{P}$ that minimizes the normalized standard deviation between the eRP and SSFM solutions of the WDM field $[18,(27)]$. Fig. 1 (bottom) shows the ratio $\hat{P} / P$ versus $D \cdot R^{2}$, which can be translated into a ratio of nonlinear phases according to (1). Such a ratio is seen to be very close to the value 2 over a wide range of $D \cdot R^{2}$ values. The factor 2 comes from the degeneracy factor of both intra- and inter-channel cross phase modulation. In dual-polarization the factor becomes $3 / 2$, as discussed in Appendix C. Note that the eRP was tacitly used in [10], [13] and related to the nonlinear constellation phase rotation in [12].

Another improvement to the basic RP1 solution is the first order logarithmic perturbation (LP) [26], [27], which approximates the electric field as $A(z, t) \simeq A_{0}(z, t)$. $\exp \left(-j A_{1}(z, t) / A_{0}(z, t)\right)$. At time instants such that $\left|A_{1}(z, t)\right|>\left|A_{0}(z, t)\right|$ the approximation may diverge, and in such a case one switches back to the RP1 solution [27]. The first order LP solution (LP1) converges to the exact one in the limit of very small dispersion; on the other hand, it is difficult to justify the statistics of the received field in the strongly dispersive regime of DU links with the LP1 solution. Numerical and experimental results showed the statistics to be circular Gaussian [1], [2] in such links. The RP1 term is the sum of many weakly correlated contributions [see (2)], and by the central limit theorem it thus has approximately Gaussian statistics. Nevertheless, Gaussian statistics for $A_{1}(z, t)$ imply log-normal statistics for the received LP1 field, which is inconsistent with experimental observations. Our results confirm that eRP is better than LP1 in predicting performance of DU links [11].

\section{A. Numerical Implementation}

There are two main different ways to write the NLI perturbation $\tilde{A}_{1}(L, \omega)$ of the RP method. The first one evaluates the integral in closed-form over the distance, leaving a double integral over the frequency domain [6], [23], [28]

$$
\begin{aligned}
\tilde{A}_{1}(L, \omega)= & -j \iint \tilde{\mathcal{K}}\left(\omega, \omega_{1}, \omega_{2}\right) \tilde{A}\left(0, \omega+\omega_{1}\right) \tilde{A}\left(0, \omega+\omega_{1}\right) \\
& \times \tilde{A}^{*}\left(0, \omega+\omega_{1}+\omega_{2}\right) \frac{\mathrm{d} \omega_{1}}{2 \pi} \frac{\mathrm{d} \omega_{2}}{2 \pi}
\end{aligned}
$$

where $\tilde{\mathcal{K}}\left(\omega, \omega_{1}, \omega_{2}\right)$ is the kernel of the optical link and we assumed, for the sake of simplicity, scalar electric fields.

The main advantage of this approach is to summarize all the properties of the optical link into a unique function, the kernel [15], which highlights the FWM nature of both intra- and interchannel nonlinear effects; the disadvantage is the complexity of the numerical evaluation of (2). If $M$ is the number of discrete frequency points, solving the double integral in (2) for each frequency calls for a $O\left(M^{3}\right)$ complexity, which can be easily unmanageable for optical links of interest.

The second approach does not close the integral over the distance $z$, expressing (2) in the following form [17], [18]

$$
A_{1}(L, t)=-j \int_{0}^{L} \gamma(z) h_{z L}(t) \otimes \mathcal{N}_{\mathrm{P}}\left(h_{0 z}(t) \otimes A(0, t)\right) \mathrm{d} z
$$

where $\otimes$ indicates convolution, $h_{s z}(t)$ is the linear impulse response from coordinate $s$ to $z$

$$
h_{s z}(t)=\mathcal{F}^{-1}\left\{\sqrt{G(s, z)} e^{-j \frac{C(s, z)}{2} \omega^{2}-j \frac{C s(s, z)}{6} \omega^{3}}\right\} .
$$

$G(s, z), C(s, z), C_{\mathrm{s}}(s, z)$ are the cumulated gain, dispersion and slope, respectively, between coordinate $s$ and $z$, respectively

$$
\begin{aligned}
& G(s, z)=e^{\int_{s}^{z}\left(-\alpha(x)+\sum_{k} \log \left(G_{k}\right) \delta\left(x-z_{k}\right)\right) \mathrm{d} x} \\
& C(s, z)=\int_{s}^{z} \beta_{2}(x) \mathrm{d} x, \quad C_{\mathrm{s}}(s, z)=\int_{s}^{z} \beta_{3}(x) \mathrm{d} x .
\end{aligned}
$$

$G_{k}$ is the power gain of the amplifier placed at coordinate $z_{k}$; $\delta(x)$ is the Dirac's delta function; $\alpha(x), \beta_{2}(x), \beta_{3}(x)$ are the fiber attenuation, dispersion and third order dispersion at coordinate $x$, respectively. Note in particular that $C(z, s)=-C(s, z)$. $\mathcal{N}_{\mathrm{P}}$ is a nonlinear operator, defined as

$$
\mathcal{N}_{\mathrm{P}}(U)=\left(|U|^{2}-2 P(z)\right) U
$$

with $P(z)=P \cdot G(0, z)$. Equation (5) defines the eRP local nonlinear operator, which in the special case of $P=0$ becomes the operator $\mathcal{N}_{0}=|U|^{2} U$ of the classical RP method; on a global scale one can simply move from RP to eRP as described in Appendix A. A direct comparison of (3) and (2) reveals that assuming ${ }^{2} \tilde{h}_{0 L}(\omega)=\mathcal{F}\left\{h_{0 L}(t)\right\}=1$, and with third order dispersive effects, the kernel is [12], [14], [23]

$$
\tilde{\mathcal{K}}=\int_{0}^{L} \gamma(z) G(0, z) e^{j \omega_{1} \omega_{2}\left[C(0, z)+C_{\mathrm{S}}(0, z)\left(\omega+\frac{\omega_{1}+\omega_{2}}{2}\right)\right]} \mathrm{d} z .
$$

Equation (3) is more efficient than (2) for numerical evaluation, since the convolutions can be efficiently performed by Fast Fourier Transforms (FFT) in $O(M \log M)$ operations [15]. Assuming a uniform discretization grid $\Delta z$ for the integral in $z$, an algorithm solving (3) is described in Fig. 2(b) as a concatenation of nonlinear $(\mathrm{N})$ and linear $(\mathrm{L})$ blocks. The nonlinear block implements $-j \gamma \mathcal{N}_{\mathrm{P}}(U) \Delta z$, while the linear block $h_{s z}(t)$ with $z=s+\Delta z$.

The "Parallel RP1" scheme [17], [18] exploits the integration in $z$ over parallel paths, a feature that can speed up the computation if a parallel simulation architecture is available [17], [29]. Please note that in each path only one nonlinear block is visited, according to the first order perturbation theory.

For comparison, in Fig. 2(a) we also report the standard SSFM. It is worth noting that the SSFM accounts for all-order nonlinearity by a nonlinear block $\mathcal{N}_{\mathrm{S}}(U)=U$. $\exp \left(-j \gamma|U|^{2} L_{\mathrm{eff}}(\Delta z)\right)$.

\footnotetext{
${ }^{2}$ This assumption corresponds to having a post-compensating fiber at $z=L$ recovering all linear impairments of a transparent link.
} 
(a) Standard SSFM

$$
A(0, t) \rightarrow \begin{array}{l|l|l|l|l|l|l|l|l|l|l|l|l|}
\hline N_{s} & L & N_{s} & L \\
\hline
\end{array}
$$

(b) Parallel RP1

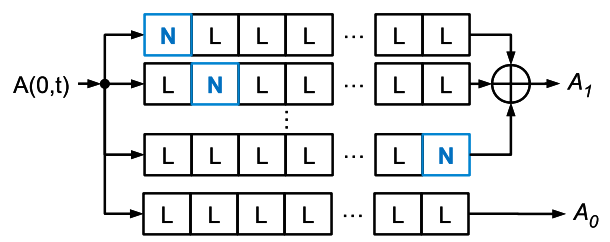

Fig. 2. SSFM and RP1 algorithm. $\mathrm{N}, \mathrm{N}_{\mathrm{S}}$ : block implementing Kerr effect. L: block implementing linear effects. An FFT/IFFT is performed at the input/output of each cascade of linear blocks.

\section{Alternative DeRIVATION OF THE GNRF}

Aim of this section is to evaluate the PSD of $A_{1}(L, t)$, which is the Fourier transform of the auto-correlation function (ACF). We proceed assuming a scalar electric field. Extension to the dual-polarization case is discussed in Appendix C. We start recalling a property of linear systems.

Lemma 1: Given two jointly stationary stochastic processes $A(t)$ and $B(t)$ having cross-correlation $R_{A B}(\tau)=$ $E\left[A(t+\tau) B^{*}(t)\right]$, the cross-correlation function of

$$
C(t)=h_{s z}(t) \otimes A(t)
$$

and

$$
D(t)=h_{l d}(t) \otimes B(t)
$$

is:

$$
R_{s z l d}(\tau)=E\left[C(t+\tau) D^{*}(t)\right]=h_{s z l d}(\tau) \otimes R_{A B}(\tau)
$$

where

$$
h_{s z l d}(\tau) \triangleq h_{s z}(\tau) \otimes h_{l d}^{*}(-\tau)
$$

Proof: See [30, Example 10-20, pp. 318-319].

We extensively use Lemma 1 to relate the ACF of the received perturbation (3) to the ACF of the input field. The result is expressed by the following theorem:

Theorem 2: If $A(0, t)$ is a stationary circular complex Gaussian process, the ACF of the eRP first-order perturbation $A_{1}(L, t)$, defined by $R_{L}(\tau)=E\left[A_{1}(L, t+\tau) A_{1}^{*}(L, t)\right]$, is

$$
\begin{aligned}
R_{L}(\tau)= & 2 \int_{0}^{L} \int_{0}^{L} \gamma(z) \gamma(s) \\
& \times h_{z L s L}(\tau) \otimes \mathcal{N}_{0}\left(h_{0 z 0 s}(\tau) \otimes R_{0}(\tau)\right) \mathrm{d} z \mathrm{~d} s
\end{aligned}
$$

with $R_{0}(\tau)=E\left[A(0, t+\tau) A^{*}(0, t)\right]$ the input ACF.

Proof: Using Lemma 1 we can write the ACF of $A_{1}(L, t)$ as:

$R_{L}(\tau)=\int_{0}^{L} \int_{0}^{L} \gamma(z) \gamma(s) h_{z L}(\tau) \otimes h_{s L}^{*}(-\tau) \otimes R_{\mathrm{N}}(z, s, \tau) \mathrm{d} z \mathrm{~d} s$

where

$$
R_{\mathrm{N}}(z, s, \tau)=E\left[\mathcal{N}_{\mathrm{P}}(U(z, t+\tau)) \mathcal{N}_{\mathrm{P}}^{*}(U(s, t))\right]
$$

with $U(z, t)=h_{0 z}(t) \otimes A(0, t)$. If we used the standard RP nonlinear operator $\mathcal{N}_{0}$ in (9) we would simply have

$R_{\mathrm{N}}^{\mathrm{RP}}(z, s, \tau)=E\left[|U(z, t+\tau)|^{2} U(z, t+\tau)|U(s, t)|^{2} U^{*}(s, t)\right]$

which involves 6 th order moments. Since, by hypothesis, $A(0, t)$ is a complex Gaussian process, the real and imaginary parts of $U(z, t+\tau)$ and $U(s, t)$ follow a multivariate Gaussian distribution, being related to $A(0, t)$ through a linear operation. In this framework the ACF in (10) can be evaluated using the complex Gaussian moment theorem [31], obtaining:

$$
R_{\mathrm{N}}^{\mathrm{RP}}(z, s, \tau)=4 R_{z z}(0) R_{s s}(0) R_{z s}(\tau)+2\left|R_{z s}(\tau)\right|^{2} R_{z s}(\tau)
$$

where $R_{z s}(\tau)=E\left[U(z, t+\tau) U^{*}(s, t)\right]$. The first term in (11) corresponds in the scalar case to the last term in [12], (26)]. As shown in Appendix B, when using instead the eRP nonlinear operator $\mathcal{N}_{\mathrm{p}}$ in (9) the first term in (11) vanishes, so that:

$$
R_{\mathrm{N}}(z, s, \tau)=2\left|R_{z s}(\tau)\right|^{2} R_{z s}(\tau)=2 \mathcal{N}_{0}\left(R_{z s}(\tau)\right)
$$

which shows that the best eRP power $\hat{P}$ discussed in Section II is such as to eliminate any dependence on $R_{z z}(0)$ and $R_{s s}(0)$ in (11).

Using Lemma 1 we have:

$$
R_{\mathrm{N}}(z, s, \tau)=2 \mathcal{N}_{0}\left(h_{0 z}(\tau) \otimes h_{0 s}^{*}(-\tau) \otimes R_{0}(\tau)\right) .
$$

Using definition (7) and Theorem 2 we finally have (8).

The Fourier transform of (8) is a generalization of the GNRF in [10], valid for any optical link. It can be further simplified by introducing the dispersive filters:

$$
\begin{aligned}
d_{s z}(\tau) & =\mathcal{F}^{-1}\left\{e^{-j \frac{C(s, z)}{2} \omega^{2}-j \frac{C_{s}(s, z)}{6} \omega^{3}}\right\} \\
d_{z s l d}(\tau) & =d_{z s}(\tau) \otimes d_{l d}^{*}(-\tau)
\end{aligned}
$$

obtaining

$$
\begin{aligned}
R_{L}(\tau)= & 2 \int_{0}^{L} \int_{0}^{L} \gamma(z) \gamma(s) \sqrt{G(z, L) G(s, L) G^{3}(0, z) G^{3}(0, s)} \\
& \times d_{z L s L}(\tau) \otimes \mathcal{N}_{0}\left(d_{0 z 0 s}(\tau) \otimes R_{0}(\tau)\right) \mathrm{d} z \mathrm{~d} s \\
= & 2 \int_{0}^{L} \int_{0}^{L} \gamma(z) \gamma(s) G(0, z) G(0, s) \\
& \times d_{z s}(\tau) \otimes \mathcal{N}_{0}\left(d_{s z}(\tau) \otimes R_{0}(\tau)\right) \mathrm{d} z \mathrm{~d} s
\end{aligned}
$$

where we used the assumption that the optical link is transparent, i.e., $G(0, z) G(z, L)=1$ and the properties $\tilde{d}_{z L s L}(\omega)=\tilde{d}_{z s}(\omega)$ and $\tilde{d}_{0 z 0 s}(\omega)=\tilde{d}_{s z}(\omega)$. The factor of 2 in (13) must be replaced by $16 / 27$ if the dual-polarization Manakov equation is used with uncorrelated polarizations, as explained in Appendix C.

We now explore some cases of interest.

\section{A. Single Span}

A single span is a fiber of length $L$ followed by an ideal optical amplifier that recovers the fiber loss. The presence of a pre- or post-compensating fiber does not have any impact, since a unitary linear operation cannot change the statistics of a circular GN. Assuming constant parameters along distance, we 
have

$$
\begin{aligned}
G(0, z) G(0, s) & =e^{-\alpha(z+s)} \\
d_{s z}(\tau) & =d_{0, z-s}(\tau)
\end{aligned}
$$

and

$$
\tilde{d}_{0 x}(\omega)=e^{-j \frac{\beta_{2}}{2} \omega^{2} x-j \frac{\beta_{3}}{6} \omega^{3} x} .
$$

With the change of variables $x=z-s$ and $y=z+s$, (13) becomes

$$
R_{L}(\tau)=\gamma^{2} \int_{-L}^{L} d_{x 0}(\tau) \otimes \mathcal{N}_{0}\left(d_{0 x}(\tau) \otimes R_{0}(\tau)\right) \int_{\Lambda} e^{-\alpha y} \mathrm{~d} y \mathrm{~d} x
$$

where the domain $\Lambda$ of integration is

$$
\Lambda(x, y)=\{x, y \in \mathbb{R}: \quad|x|<y<2 L-|x|\} .
$$

The integral in $y$ can be solved in closed form, yielding

$$
f(x) \triangleq \int_{|x|}^{2 L-|x|} e^{-\alpha y} \mathrm{~d} y= \begin{cases}\frac{2 e^{-\alpha L} \sinh (\alpha(L-|x|))}{\alpha}, & \alpha \neq 0 \\ 2(L-|x|), & \alpha=0 .\end{cases}
$$

In particular, we have the following asymptotic

$$
\lim _{L \rightarrow \infty} f(x)=\frac{e^{-\alpha x}}{\alpha} .
$$

For a single span the identity $d_{-s,-z}(\tau)=d_{s z}^{*}(-\tau)$ holds. Introducing the function

$$
\tilde{G}_{0 x}(\omega) \triangleq \mathcal{F}\left\{d_{x 0}(\tau) \otimes \mathcal{N}_{0}\left(d_{0 x}(\tau) \otimes R_{0}(\tau)\right)\right\}
$$

we observe that $\tilde{G}_{0,-x}(\omega)=\tilde{G}_{0, x}^{*}(\omega)$ since $R_{0}(\tau)$ has Hermitian symmetry, i.e., $R_{0}(\tau)=R_{0}^{*}(-\tau)$, and $\mathcal{N}_{0}\left(A^{*}\right)=$ $\left(\mathcal{N}_{0}(A)\right)^{*}$. Exploiting this symmetry with the even symmetry of $f(x)$, the PSD of the NLI noise can be written as

$$
\tilde{G}_{L}(\omega) \triangleq \mathcal{F}\left\{R_{L}(\tau)\right\}=2 \gamma^{2} \int_{0}^{L} \Re\left[\tilde{G}_{0 x}(\omega)\right] f(x) \mathrm{d} x
$$

with $\Re\{$.$\} the real part.$

It is worth noting that the double integral in (13) has been reduced to a single integral, with great savings in numerical computation.

\section{B. Homogeneous Multi-Span}

The optical link is here composed of $N$ identical spans, each of length $z_{\mathrm{A}}$, with dispersion and gain map shown in Fig. 3. The transmission fibers have dispersion $D$ while the in-line residual dispersion per span is $D_{\text {in }}$. DU links are a special case with $D_{\text {in }}=D \cdot z_{\mathrm{A}}$. It is helpful to split the integrals as follows

$$
\begin{aligned}
R_{L}(\tau)= & 2 \gamma^{2} \sum_{k, n=0}^{N-1} \int_{k z_{\mathrm{A}}}^{(k+1) z_{\mathrm{A}}} \int_{n z_{\mathrm{A}}}^{(n+1) z_{\mathrm{A}}} G(0, z) G(0, s) \\
& \times d_{z s}(\tau) \otimes \mathcal{N}_{0}\left(d_{s z}(\tau) \otimes R_{0}(\tau)\right) \mathrm{d} z \mathrm{~d} s
\end{aligned}
$$

where $L=N \cdot z_{\mathrm{A}}$ is the link length. Introducing the local variables $\xi=z-k z_{\mathrm{A}}$ and $\nu=s-n z_{\mathrm{A}}$, by observing Fig. 3 we
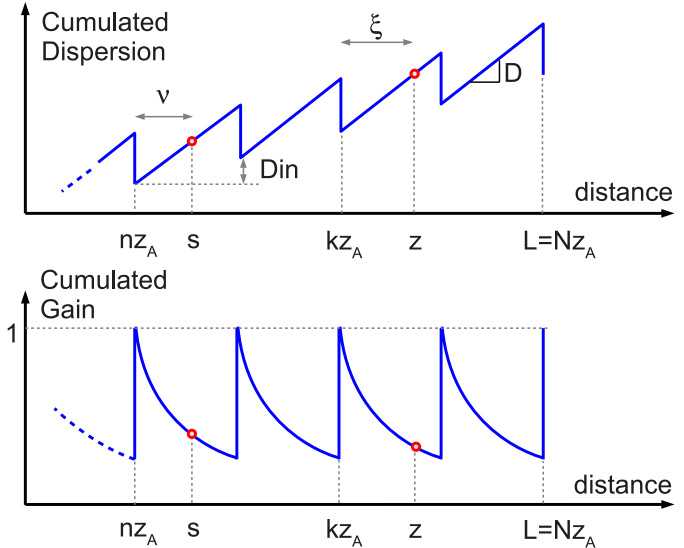

Fig. 3. Dispersion map (top) and gain map (bottom) of an homogeneous periodic optical link.

note the following

$$
\begin{aligned}
G(0, z) G(0, s) & =e^{-\alpha(\xi+\nu)} \\
d_{s z}(\tau) & =d_{0,(k-n) \zeta_{\text {in }}+\xi-\nu}(\tau)
\end{aligned}
$$

where $\zeta_{\text {in }}=D_{\text {in }} / D$ is the coordinate after which a dispersion of $D_{\text {in }}$ has been cumulated ${ }^{3}$. With the change of variables

$$
x=\xi-\nu, \quad y=\xi+\nu, \quad m=k-n
$$

we have

$$
\begin{aligned}
R_{L}(\tau)= & \gamma^{2} \sum_{m=-(N-1)}^{N-1}(N-|m|) \int_{-z_{\mathrm{A}}}^{z_{\mathrm{A}}} d_{m} \zeta_{\text {in }}+x, 0 \\
& \otimes \mathcal{N}_{0}\left(d_{0, m \zeta_{\text {in }}+x}(\tau) \otimes R_{0}(\tau)\right) \int_{\Lambda} e^{-\alpha y} \mathrm{~d} y \mathrm{~d} x .
\end{aligned}
$$

Following the same steps leading to (19) we finally have the GNRF for multi-span systems as

$$
\tilde{G}_{L}(\omega)=2 \gamma^{2} \sum_{m=-(N-1)}^{N-1}(N-|m|) \int_{0}^{z_{\mathrm{A}}} \Re\left[\tilde{G}_{0, m \zeta_{\text {in }}+x}(\omega)\right] f(x) \mathrm{d} x
$$

where: $f(x)$ is defined in (16) and here it has to be used with $z_{\mathrm{A}}$ in place of $L ; \tilde{G}_{0 x}(\omega)$ is defined in (18). Since $\tilde{G}_{0 x}(\omega)$ also writes as

$$
\begin{aligned}
\tilde{G}_{0 x}= & \int_{-\infty}^{\infty} \int_{-\infty}^{\infty} e^{j \omega_{1} \omega_{2}\left[C(0, x)+C_{\mathrm{S}}(0, x)\left(\omega+\frac{\omega_{1}+\omega_{2}}{2}\right)\right]} \\
& \times \tilde{G}_{0}\left(\omega+\omega_{1}\right) \tilde{G}_{0}\left(\omega+\omega_{2}\right) \tilde{G}_{0}\left(\omega+\omega_{1}+\omega_{2}\right) \frac{\mathrm{d} \omega_{1}}{2 \pi} \frac{\mathrm{d} \omega_{2}}{2 \pi}
\end{aligned}
$$

where $\tilde{G}_{0}(\omega)=\mathcal{F}\left\{R_{0}(\tau)\right\}$, for DU links without 3rd order dispersion, after some algebra, (21) becomes [10, (1)].

Equation (21) shows that the integral is limited to $\left(0, z_{\mathrm{A}}\right)$. Nevertheless, each term of the sum is properly weighted by $N-|m|$. For instance, when $m=0$ we have $N$ equal contributions, each corresponding to a particular span of the link. These particular contributions are all equal because the cumulated dispersion between the beginning of the optical link

\footnotetext{
${ }^{3}$ In particular, in DU links it is $\zeta_{\text {in }}=z_{\mathrm{A}}$.
} 


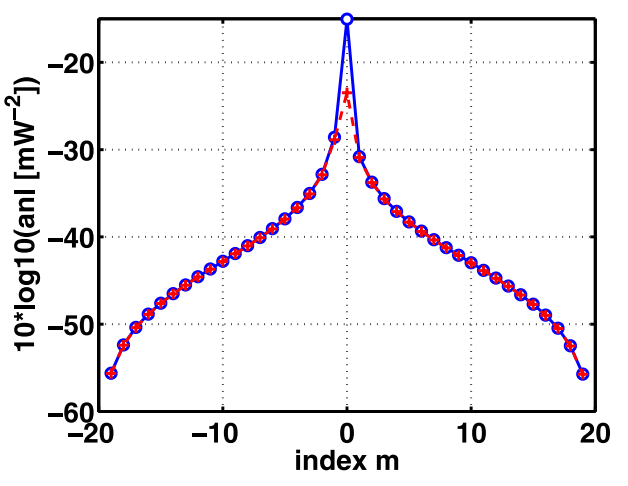

Fig. 4. $\quad a_{\mathrm{NL}}$ coefficient $[\mathrm{dB}]$ versus index $m$ of (21). The summation of all points of the figure gives the final $a_{\mathrm{NL}}$. (Solid with "०"): 15 channels. (Dashed with "+"): 1 channel. SMF, $20 \times 100$ km DU link. $R=25$ Gbaud.

and the beginning of the generic span does not change the statistics of a circular GN, and thus is irrelevant. The terms weighted by $m=1$ account for the $N-1$ combinations of cross-correlations between two neighboring spans, and so on. The author in [10] already reached similar conclusions, calling the $m=0$ and $m \neq 0$ terms as incoherent and coherent noise accumulation terms, respectively.

The largest contribution to the sum in (21) comes for $m=0$, as shown in Fig. 4 for both a single- and 15-channel Nyquist WDM system with polarization division multiplexing (PDM) signals of uncorrelated and statistically identical $\mathrm{X}$ and $\mathrm{Y}$. The figure shows $a_{\mathrm{NL}}[\mathrm{dB}]$, defined as the NLI variance $(\mathrm{X}+\mathrm{Y})$ per unit power [6], evaluated for each value of $m$ in (21) in a $20 \times 100 \mathrm{~km}$ single-mode fiber $(\mathrm{SMF}) \operatorname{link}(D=17 \mathrm{ps} / \mathrm{nm} / \mathrm{km})$. Note that the shape of $a_{\mathrm{NL}}$ is almost of even symmetry, but not exactly. The reason is related to the fact that $\tilde{G}_{0, m \zeta_{\text {in }}+x}(\omega)$ is not an even function of $m$, but the symmetry is satisfied as $m \rightarrow \infty$.

Equation (21) highlights the fact that the scaling law of the NLI PSD with the number of spans $N$ is not linear [10], [32]. Assuming a linear scaling actually corresponds to neglecting all span cross-contributions $m \neq 0$. According to Fig. 4, such contributions are negligible in WDM transmission, where the $m=0$ term is by far dominant, but not in single channel transmission, in agreement with what observed in [10], [32]. We will return to this point in Section IV.

\section{Example: Gaussian Spectrum}

The convolutions in (18) can be evaluated in closed form for a Gaussian shaped spectrum and neglecting fiber's third order dispersion. A Gaussian function is form invariant under the Fourier transform

$$
\eta_{\omega}\left(\sigma^{2}\right) \triangleq e^{-\frac{\omega^{2}}{2 \sigma^{2}}} \stackrel{\mathcal{F}^{-1}}{\longleftrightarrow} \sqrt{\frac{\sigma^{2}}{2 \pi}} e^{-\frac{\tau^{2} \sigma^{2}}{2}} \equiv \sqrt{\frac{\sigma^{2}}{2 \pi}} \eta_{\tau}\left(\frac{1}{\sigma^{2}}\right) .
$$

We derive a closed form expression of (18) by mimicking the steps of an algorithm. Hence, first we perform the inner linear filtering, then the nonlinear operator, finally the inverse GVD filtering. Starting from $\mathcal{F}\left\{R_{0}(\tau)\right\}=\eta_{\omega}\left(\sigma^{2}\right)$, after each step the PSD is:
1) After inner linear filter (which also defines $B$ )

$$
\eta_{\omega}\left(\frac{\sigma^{2}}{1+j \sigma^{2} \beta_{2} x}\right)=\eta_{\omega}(B)
$$

2) Returning back to time

$$
\sqrt{\frac{B}{2 \pi}} \eta_{\tau}\left(\frac{1}{B}\right)
$$

3) $\operatorname{After} \mathcal{N}_{0}($.

$$
\frac{B}{2 \pi} \sqrt{\frac{B^{*}}{2 \pi}} \eta_{\tau}\left(\frac{1}{2 B+B^{*}}\right)
$$

4) Returning back to frequency

$$
\frac{B}{2 \pi} \sqrt{\frac{B^{*}}{2 \pi}} \sqrt{\frac{2 \pi}{2 B+B^{*}}} \eta_{\omega}\left(2 B+B^{*}\right)
$$

5) After outer linear filter

$$
\tilde{G}_{0 x}(\omega)=\frac{B \sqrt{B^{*}}}{2 \pi \sqrt{2 B+B^{*}}} \eta_{\omega}\left(\frac{2 B+B^{*}}{1-\left(2 B+B^{*}\right) j \beta_{2} x}\right) .
$$

Substituting the definition of $B$ given at point 2) in the final expression we have

$$
\tilde{G}_{0 x}(\omega)=\frac{\sigma^{2} e^{-\frac{\omega^{2}}{2 \sigma^{2}} \frac{1-j 3 \beta_{2} x \sigma^{2}}{3-j \beta_{2} x \sigma^{2}}}}{2 \pi \sqrt{3+j 2 \beta_{2} x \sigma^{2}+\beta_{2}^{2} x^{2} \sigma^{4}}} .
$$

Substitution in (21) yields the final PSD in single-integral form, which is very fast to numerically evaluate. For instance, such an expression can be used to double-check the more general algorithm proposed in this paper for debugging purposes. The Gaussian spectrum can also be used to infer the NLI PSD in presence of a very wide input WDM spectrum without resorting to FFT. To this purpose, we relate $\sigma$ to the bandwidth $B_{\text {WDM }}$ of the WDM transmission having the same NLI variance at the system output. We evaluated 300 different systems, whose random parameters were uniformly chosen in the following range: number of channels (1 to 31 ); number of spans (5 to 100); symbol rate (5 to 28 Gbaud); fiber dispersion (2 to $30 \mathrm{ps} / \mathrm{nm} / \mathrm{km}$ ). Each system was a Nyquist WDM, whose central channel was detected through a matched filter. We found that $\sigma / B_{\mathrm{WDM}}=8.16$ gives a best NLI variance fit, with error within $0.1 \mathrm{~dB}$ of the true value when the number of channels is larger than 10 .

\section{ASYMPTOTIC EXPANSION}

In the previous equations we faced convolutions with dispersive filters like $\tilde{d}_{0 x}(\omega)$ in (14). When the cumulated dispersion is large, the dispersion makes the phase of $\tilde{d}_{0 x}(\omega)$ oscillate very fast with $\omega$, a condition that suggests to use the saddlepoint approximation to approximate the linear convolution. Such approximation is the topic of this section. We start by briefly recalling without proof the basic result of the saddlepoint approximation, which for a purely oscillating function is also called the method of the stationary phase [22].

Theorem 3 (Stationary phase approximation (SPA)): Assume that the real function $\phi(\omega)$ has a unique saddlepoint $\omega_{0}$ such 
that $\phi^{\prime}\left(\omega_{0}\right)=0$. The integral

$$
r(\kappa)=\int_{-\infty}^{\infty} G(\omega) e^{j \kappa \phi(\omega)} \frac{\mathrm{d} \omega}{2 \pi}
$$

has the asymptotic behavior

$$
r(\kappa) \sim \frac{e^{j\left[\kappa \phi\left(\omega_{0}\right)+\operatorname{sgn}\left(\phi^{\prime \prime}\left(\omega_{0}\right)\right) \frac{\pi}{4}\right]}}{\sqrt{2 \pi \kappa\left|\phi^{\prime \prime}\left(\omega_{0}\right)\right|}} G\left(\omega_{0}\right), \quad \kappa \rightarrow \infty .
$$

Note that the theorem requires $\kappa \rightarrow \infty$ to hold, which corresponds to assuming $G(\omega)$ is slowly varying compared to the oscillations of the phase. This requirement is generally not satisfied by the Fourier transform of an electric field, which is normally extremely nervous with fast variations; on the other hand, a PSD is normally slowly varying since it averages out the mentioned variations of the Fourier transform according to the periodogram interpretation.

Our target is to evaluate (21) using the SPA. For the sake of simplicity we neglect third order dispersion. The problem is thus to evaluate

$$
\tilde{G}_{0 s}(\omega)=\mathcal{F}\left\{d_{s 0}(\tau) \otimes \mathcal{N}_{0}\left(d_{0 s}(\tau) \otimes R_{0}(\tau)\right)\right\} .
$$

We start by the inner convolution within the nonlinear operator

$$
P_{s}(\tau)=d_{0 s}(\tau) \otimes R_{0}(\tau)=\int_{-\infty}^{\infty} \tilde{G}_{0}(\omega) e^{-j \frac{\beta_{2}}{2} \omega^{2} s+j \omega \tau} \frac{\mathrm{d} \omega}{2 \pi} .
$$

Here $\phi(\omega)=-\frac{\beta_{2}}{2} \omega^{2} s+\omega \tau$, thus the saddlepoint is

$$
\phi^{\prime}\left(\omega_{0}\right)=0 \Rightarrow \omega_{0}=\frac{\tau}{\beta_{2} s} .
$$

We further have:

$$
\phi\left(\omega_{0}\right)=\frac{1}{2} \frac{\tau^{2}}{\beta_{2} s}, \quad \phi^{\prime \prime}\left(\omega_{0}\right)=-\beta_{2} s .
$$

Equation (23) using the SPA becomes:

$$
P_{s}(\tau) \simeq \tilde{G}_{0}\left(\frac{\tau}{\beta_{2} s}\right) \frac{e^{j \frac{\tau^{2}}{2 \beta_{2} s}-j \operatorname{sgn}\left(\beta_{2} s\right) \frac{\pi}{4}}}{\sqrt{2 \pi\left|\beta_{2} s\right|}} .
$$

The variable $s$ has to be replaced with $m \zeta_{\text {in }}+x$ to evaluate (21). It turns out that for the SPA to hold, we need $\left|\beta_{2}\left(m \zeta_{\text {in }}+x\right)\right| \gg 1$, which, for instance, is unsatisfied for $x \simeq 0$ and $m=0$.

After the nonlinear step we have:

$$
\mathcal{N}_{0}\left(P_{s}(\tau)\right) \simeq \tilde{G}_{0}^{3}\left(\frac{\tau}{\beta_{2} s}\right) \frac{e^{j \frac{\tau^{2}}{2 \beta_{2} s}-j \operatorname{sgn}\left(\beta_{2} s\right) \frac{\pi}{4}}}{\left(2 \pi\left|\beta_{2} s\right|\right)^{3 / 2}} .
$$

To complete the evaluation we have to move to the frequency domain and apply the inverse dispersion. This step is exactly the undo of the step that leads to (24). Moreover, since (25) has the same functional behavior of (24), we infer that undoing the dispersion using the SPA yields:

$$
\tilde{G}_{0 s}(\omega) \simeq \frac{\tilde{G}_{0}^{3}(\omega)}{2 \pi\left|\beta_{2} s\right|}
$$

Returning back to the original variable:

$\tilde{G}_{0, m \zeta_{\text {in }}+x}(\omega) \simeq \frac{\tilde{G}_{0}^{3}(\omega)}{2 \pi\left|\beta_{2}\left(m \zeta_{\text {in }}+x\right)\right|}, \quad\left|\beta_{2}\left(m \zeta_{\text {in }}+x\right)\right| \gg 1$.

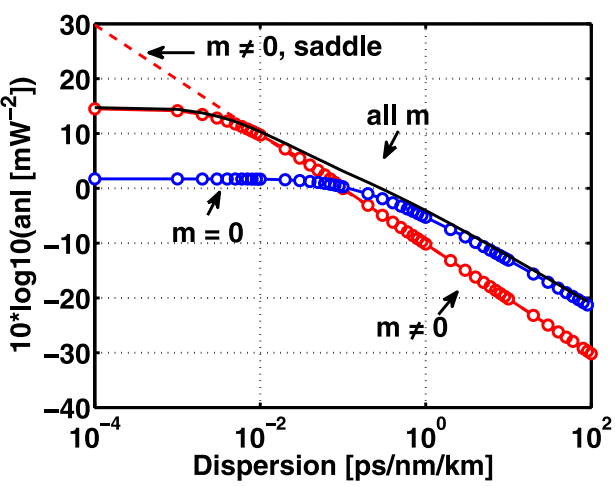

Fig. 5. Coefficient $a_{\mathrm{NL}}$ versus fiber dispersion including only selected $m$ indexes in the summation (21). The PSD $\tilde{G}_{0, m} \zeta_{\text {in }}+x(\omega)$ has been evaluated by FFT of (18) or by the stationary phase approximation (27) (dashed line). Note that for large dispersions the scaling is $1 / \beta_{2}$, according to (27). 15 channels, $R=25$ Gbaud, Nyquist WDM, DU $20 \times 100 \mathrm{~km}$ link.

Note that a dependence on $\tilde{G}_{0}^{3}(\omega)$ indicates that cross-channel effects do not affect the NLI variance, as confirmed by the coincidence of $m \neq 0$ terms in single-channel and WDM cases in Fig. 4. This way, the $m=0$ term increasingly contributes to the NLI PSD for increasing number of channels.

Equation (27) suggests a scaling rule ${ }^{4}$ with $1 / \beta_{2}$, in agreement with $[10,(20)]$. This indication is confirmed by Fig. 5, which shows $a_{\mathrm{NL}}$ versus fiber dispersion for a Nyquist WDM made of 15 channels at $R=25$ Gbaud each. As expected, the SPA (dashed line) holds at not too small dispersions, where it instead predicts a divergence of $\tilde{G}_{0, m \zeta_{\text {in }}+x}(\omega)$ that does not match the real behavior. It is worth noting that the term $m=0$ dominates at large dispersions, even if the contribution of $m \neq 0$ terms cannot be neglected [32] (e.g., "all m" curve and $m=0$ curve differ by $0.7 \mathrm{~dB}$ at $D=17 \mathrm{ps} / \mathrm{nm} / \mathrm{km}$ ). Even if not shown here, the cross-point between the $m=0$ and $m \neq 0$ curves moves at higher dispersions for decreasing $R$ (e.g., $2 \mathrm{ps} / \mathrm{nm} / \mathrm{km}$ at $R=5$ Gbaud).

Using (27) and (17) it is possible to simplify (21) for $m \neq 0$ in the limit $\alpha\left|m \zeta_{\text {in }}\right|>\alpha z_{\mathrm{A}} \gg 1$. Calling this $\operatorname{PSD} \tilde{G}_{L}^{\mathrm{cc}}(\omega)$, we get

$$
\begin{aligned}
\tilde{G}_{L}^{c c}(\omega) \simeq & 2 \gamma^{2} \sum_{m \neq 0}(N-|m|) \int_{0}^{z_{\mathrm{A}}} \frac{\tilde{G}_{0}^{3}(\omega) e^{-\alpha x}}{2 \pi\left|\beta_{2}\left(m \zeta_{\text {in }}+x\right) \alpha\right|} \mathrm{d} x \\
= & 2 \gamma^{2} \sum_{m \neq 0}(N-|m|) \tilde{G}_{0}^{3}(\omega) e^{\alpha m \zeta_{\text {in }}} \\
& \times \frac{\Gamma\left(0, \alpha m \zeta_{\text {in }}\right)-\Gamma\left(0, \alpha m \zeta_{\text {in }}+z_{\mathrm{A}}\right)}{2 \pi \alpha\left|\beta_{2}\right| \operatorname{sgn}\left(m \zeta_{\text {in }}\right)} \\
\simeq & 2 \gamma^{2} \frac{\tilde{G}_{0}^{3}(\omega)}{2 \pi \alpha^{2}\left|\beta_{2} \zeta_{\text {in }}\right|} \sum_{m \neq 0} \frac{(N-|m|)}{|m|}
\end{aligned}
$$

\footnotetext{
${ }^{4}$ This scaling rule is of concern in the numerical solution of (21) using typical quadrature routines at fixed absolute tolerance. For instance, although $a \int \frac{f(x)}{a} \mathrm{~d} x=\int f(x) \mathrm{d} x$, the number of function evaluations done by quad. $m$ in MATLAB depends on the value of $a$. Hence, we suggest to scale $R_{0}(\tau)$ by $\left|\beta_{2}\right|^{1 / 3}$ before calling a quadrature routine, and to undo the scaling after the call.
} 


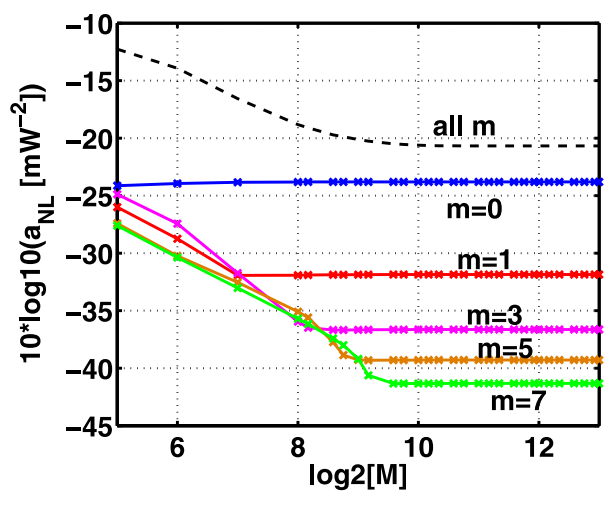

Fig. 6. Top: $a_{\mathrm{NL}}$ versus FFT size $M$ at different index $m$ of the summation in (21). Single channel, $R=28$ Gbaud, SMF $20 \times 100 \mathrm{~km}$ DU link.

where in the last step we used the asymptotic expansion of the incomplete Gamma function, i.e., $\Gamma(0, z) \sim e^{-z} / z$. Equation (28) matches with $[10,(20)]^{5}$ after extending it to dual-polarization (see Appendix C), except that (28) applies to all $\omega$, not only at $\omega=0$ as in $[10,(20)]$. We verified that for a $R=25$ Gbaud 15-channel PDM Nyquist WDM system propagating into a SMF $20 \times 100 \mathrm{~km}$ DU link, the last approximation in (28) yields an error smaller than $0.2 \mathrm{~dB}$ on $a_{\mathrm{NL}}$ compared to direct evaluation of the integral. We can conclude that, under the hypotheses of (28), the cross-correlation between spans scales as the inverse of the cumulated dispersion between the spans.

In the opposite limit of $\left|\beta_{2}\left(m \zeta_{\text {in }}+x\right)\right| \ll 1$ one can still use the SPA, but in the time domain. In this case one simply obtains $P_{s}(\tau) \simeq R_{0}(\tau)$, as it can be inferred directly by the observation that in such a case $d_{0 s}(\tau) \simeq \delta(\tau)$. This way $R_{0 s}(\tau)=R_{0}^{3}(\tau)$, thus independent of dispersion, as confirmed by the flatness of the $m=0$ curve at low dispersions in Fig. 5. Hence, the SPA is able to capture the asymptotic behavior of the PSD in both small and high dispersion regimes.

\section{A. Implications on Numerical Evaluation of NLI Variance}

The SPA is actually more accurate than the direct integration through "practical" FFT for very large dispersions. The reason is related to the large window size required by the FFT, which is proportional to the product $D \cdot R^{2}$ [33]. When this number is too large, the window size is too large so that truncation is necessary, which automatically translates into aliasing. Fig. 6 explains this observation by an example in single channel PDM transmission of a rectangular transmitted spectrum. The simulation consisted of measuring the coefficient $a_{\mathrm{NL}}$ using (21) for different values of $m$ at increasing FFT window lengths. As expected, large cumulated dispersions, i.e., large $m$, call for large FFT sizes to observe convergence. On the other hand, (27) does not suffer from aliasing and improves in accuracy at increasing dispersion, making it the right choice in the strongly dispersive regime. This observation suggests to identify a proper threshold $\chi$ such that for all coordinates $x$ in (21) satisfying $\left|m \zeta_{\text {in }}+x\right|<\chi$ the convolutions in (18) are evaluated by FFT, otherwise by the asymptotic expansion (27). It turns out that the FFT must not

\footnotetext{
${ }^{5} \mathrm{~A}$ typo in $[10,(20)]$ missed the factor $G_{\mathrm{W} \mathrm{DM}}^{3}$.
}

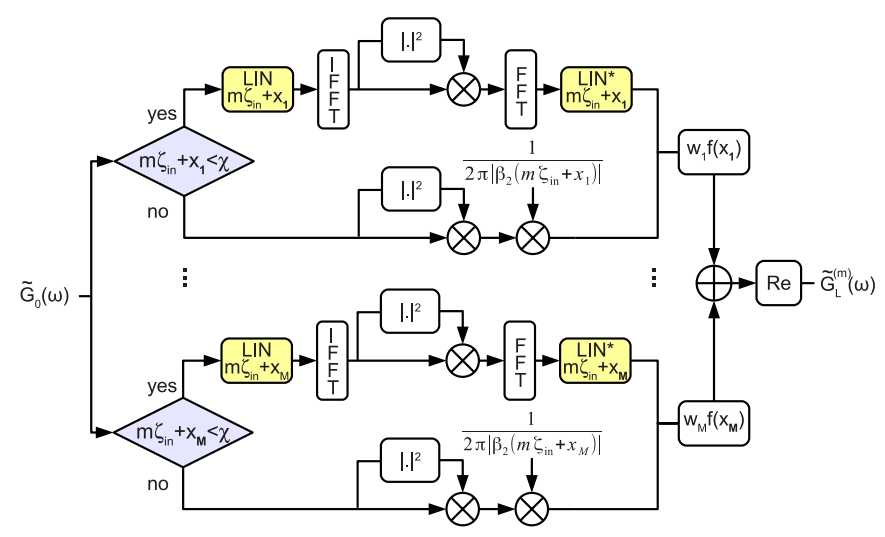

Fig. 7. Block diagram for the evaluation of (21), here expressed as $\tilde{G}_{L}(\omega)=$ $2 \gamma^{2} \sum_{m}(N-m) \tilde{G}_{L}^{(m)}(\omega)$. The integral in $z$ is discretized over $M$ nodes, weighted by coefficients $w_{k}$ according to the adopted quadrature scheme. Each FFT is sized against the cumulated dispersion at coordinate $\chi$.

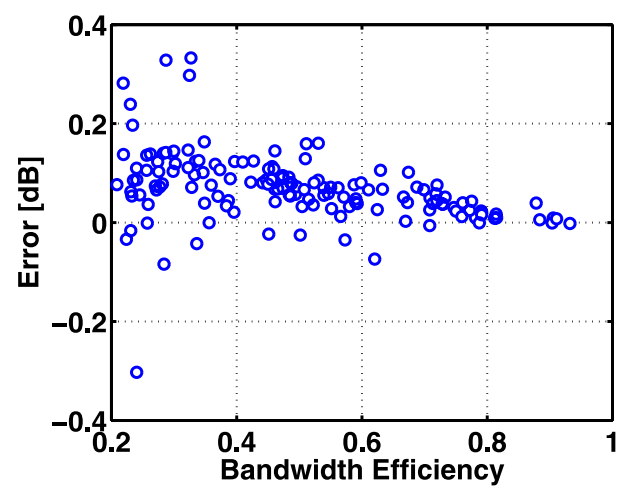

Fig. 8. Discrepancy between $a_{\mathrm{NL}}$ estimated by full FFT-based convolutions in (18) and by the proposed mixture of stationary phase approximation/FFT using a threshold equal to $|D| \chi R^{2}=10^{5} \mathrm{Gbaud}^{2} \cdot \mathrm{ps} / \mathrm{nm}$. For coordinates smaller than $\chi$ we used FFT, otherwise the stationary phase approximation. Random DU link, see text.

be sized against the worst case of cumulated dispersion, but just against the cumulated dispersion at the threshold coordinate $\chi$, with great savings in memory allocation and computational time. The general algorithm is sketched in Fig. 7. Note that when the SPA cannot be used the algorithm resembles the parallel RP1 in Fig. 2, with one nonlinear block per branch.

We estimated a good value for $\chi$ by testing 150 different DU links with uniformly distributed parameters in the range: dispersion, from 2 to $30 \mathrm{ps} / \mathrm{nm} / \mathrm{km}$; number of spans, from 1 to 60 ; roll-off, from 0 to 1 ; symbol rate, from 5 to 40 Gbaud; number of channels, from 3 to 51 ; bandwidth efficiency, defined as the ratio between the channel spacing and the symbol rate, from 0.2 to the maximum value avoiding spectral overlap. We first performed the estimation by a full FFT-based simulation with FFT bandwidth of at least three times the WDM bandwidth and frequency resolution of at least $R / 32768$. Then, we repeated the estimation with the suggested mixture of SPA/FFT algorithm for varying $\chi$. Fig. 8 shows the estimated error between the two approaches, using $|D| \chi R^{2}=10^{5} \mathrm{Gbaud}^{2} \cdot \mathrm{ps} / \mathrm{nm}$. The error is less than $0.1 \mathrm{~dB}$ in a wide range of bandwidth efficiencies, confirming that in practical situations one can safely use the 
proposed threshold to speed up computation. As a reference, evaluating $a_{\mathrm{NL}}$ as in Fig. 7 for a $50 \mathrm{GHz}$ spaced 31-channel system, with $R=25$ Gbaud, propagating either into a SMF DU $20 \times 100 \mathrm{~km}$ or $120 \times 50 \mathrm{~km}$ link took 5 s using MATLAB on a INTEL i7-3520M processor.

\section{B. Best PSD Minimizing NLI Variance}

The GN model is a simple tool to estimate the performance of a DU link, and thus can be used to optimize a transmission. Using the asymptotic expression of the PSD (27) we can prove the following theorem:

Theorem 4 (Flat is optimal): Using the asymptotic expression (27), the PSD $\tilde{G}_{0}(\omega)$ minimizing the NLI noise variance, evaluated over a frequency-bandwidth $B$ and for a fixed transmitted power $P$, is a rectangular function of width $2 \pi B$.

Proof: As per (27), the asymptotic expansion of the NLI PSD is proportional to $\tilde{G}_{0}^{3}(\omega)$. We are thus searching for $\tilde{G}_{0}(\omega)$ such that this integral is minimum

$$
\int_{-\pi B}^{\pi B} \tilde{G}_{0}^{3}(\omega) \frac{\mathrm{d} \omega}{2 \pi}
$$

subject to the input power constraint

$$
\int_{-\pi B}^{\pi B} \tilde{G}_{0}(\omega) \frac{\mathrm{d} \omega}{2 \pi}=P
$$

The solution is obtained solving the Euler-Lagrange equation for isoperimetric problems [34, p. 48]. Such equation writes as

$$
\frac{\mathrm{d} \tilde{G}_{0}^{3}}{\mathrm{~d} \tilde{G}_{0}}-\lambda \frac{\mathrm{d} \tilde{G}_{0}}{\mathrm{~d} \tilde{G}_{0}}=3 \tilde{G}_{0}^{2}-\lambda=0
$$

where $\lambda$ is the Lagrange multiplier. The solution is $\tilde{G}_{0}(\omega)=$ $\sqrt{\lambda / 3}$. Inserting in the constraint (30) we get

$$
\left.\tilde{G}_{0}(\omega)\right|_{\text {opt }}= \begin{cases}P / B & |\omega|<2 \pi B \\ 0 & \text { else }\end{cases}
$$

i.e., the best PSD is flat over the available bandwidth.

As discussed in Section IV-A, (27) does not generally hold for coordinates lower than $\chi$, corresponding to small cumulated dispersions, thus making Theorem 4 apparently weak. However, in such a regime one can use the SPA in the time domain, and obtain $R_{z}(\tau) \propto R_{0}^{3}(\tau)$, as shown at the end of Section IV. The extra contribution of this term to (29) is proportional to $\int_{-\infty}^{\infty} R_{0}^{3}(\tau) h(-\tau) \mathrm{d} \tau, h(\tau)$ being the impulse response of the detection filter, equal to $B \operatorname{sinc}(B \tau)$ in (29). Since $R_{0}(\tau)$ is the ACF of the WDM signal, thus of bandwidth much larger than $B$ for practical WDM spectra, $R_{0}(\tau)$ can safely be substituted by $R_{0}(0)$ in the previous integral. Hence, the extra term is almost independent of the shape of $R_{0}(\tau)$, thus with no impact on the functional derivative (31).

The following numerical observations corroborate Theorem 4. As in Fig. 5, we fixed the channel spacing to $25 \mathrm{GHz}$ and the channel bandwidth to the same value (Nyquist WDM for a total of 15 channels), and measured $a_{\mathrm{NL}}$ when shaping the per-channel input PSD as described in Fig. 9. The one-sided PSD was sampled at 11 equally spaced points (nodes), and then the corresponding samples were interpolated

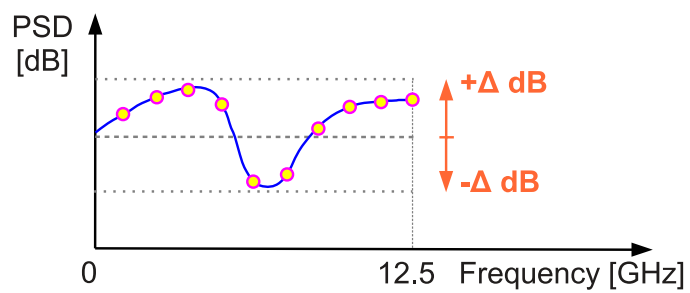

Fig. 9. Per-channel input PSD. Within the channel bandwidth we selected 11 equally spaced random values and interpolated those values to get the whole input PSD at all in-band frequencies. The maximum excursion from the flat spectrum was constrained within $\pm \Delta \mathrm{dB}$.

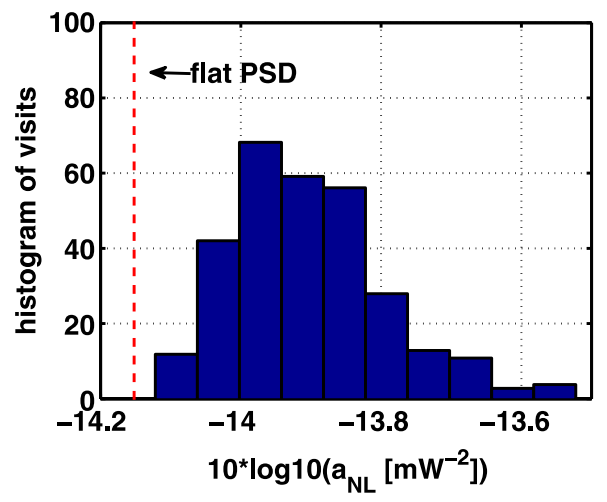

Fig. 10. histogram of $a_{\mathrm{NL}}$ using 15 channels with a SMF DU $20 \times 100 \mathrm{~km}$ link. $\Delta=3 \mathrm{~dB}$ (see Fig. 9).

by a Piecewise Cubic Hermite interpolation (function pchip of MATLAB). The two-sided PSD was of even symmetry.

Starting from a flat PSD having full-width bandwidth of $25 \mathrm{GHz}$, each node was perturbed by multiplying it by a uniformly distributed random number with a maximum excursion of $\pm 3 \mathrm{~dB}$, see Fig. 9. The per-channel power was finally restored to a fixed value $P .300$ different random seeds were tested and for each seed we measured the corresponding $a_{\mathrm{NL}}$. The lowpass filter at the receiver was in any case of rectangular shape with bandwidth $25 \mathrm{GHz}$. The histogram of the realizations of the random variable $a_{\mathrm{NL}}$ is reported in Fig. 10. None of the 300 seeds provided an $a_{\mathrm{NL}}$ value lower than that obtained with a flat PSD.

\section{CONCLUSION}

An alternative framework to derive and analyze the GN model has been proposed. We showed that the GNRF comes from a first-order eRP solution of the propagation equation, which is a first-order Volterra expansion in a suitable reference system. We provided a rigorous alternative derivation of the GNRF by working in the time domain. The new proof has been exploited to build an efficient algorithm for the numerical evaluation of the NLI noise variance for any input PSD.

We also explored an asymptotic expansion of the GNRF, showing that it can be safely used in a strongly dispersive scenario. Such an approximation has interesting physical consequences. For instance, using it we are able to prove that the best input PSD minimizing the NLI noise in a fixed bandwidth and constrained to a fixed power is the flat spectrum. 
Finally, in Appendix $\mathrm{C}$ we proved the extension of the GNRF to dual polarization including possible correlations between the polarization tributaries.

\section{APPENDIX A}

\section{RELATION BETWEEN ERP AND RP}

In this Appendix we relate the eRP solution to the RP one, showing that it is possible to move from one solution to the other by a simple transformation. For the sake of simplicity we refer to single polarization transmission, but the result holds even in PDM. According to [18], the 0th term of RP expansion (unperturbed solution) is

$$
A_{0}(z, t)=h_{0 z}(t) \otimes A(0, t) .
$$

Using (3) we have

$$
A_{1}(L, t)=-j \int_{0}^{L} \gamma(z) h_{z L}(t) \otimes \mathcal{N}_{\mathrm{P}}\left(A_{0}(z, t)\right) \mathrm{d} z
$$

where $\mathcal{N}_{\mathrm{p}}$ is defined in (5). The RP however uses $\mathcal{N}_{0}$ as the nonlinear operator, hence

$$
\begin{aligned}
& A_{1}^{\mathrm{RP}}(z, t)=-j \int_{0}^{L} \gamma(z) h_{z L}(t) \otimes\left(\left|A_{0}(z, t)\right|^{2} A_{0}(z, t)\right) \mathrm{d} z \\
& A_{1}^{\mathrm{ePP}}(z, t)=A_{1}^{\mathrm{RP}}+j \int_{0}^{L} \gamma(z) h_{z L}(t) \otimes\left(P(z) A_{0}(z, t)\right) \mathrm{d} z
\end{aligned}
$$

where $P(z)=P \cdot G(0, z)$ was defined in Section. II-A. Since $h_{z L}(t) \otimes h_{0 z}(t)=h_{0 L}(t)$ we finally have

$$
A_{1}^{\mathrm{eRP}}(L, t)=A_{1}^{\mathrm{RP}}(L, t)+j A_{0}(L, t) \int_{0}^{L} \gamma(z) P(z) \mathrm{dz} .
$$

For a periodic system of $N$ spans the last integral is $\Phi_{\mathrm{NL}}(P)$.

\section{APPENDIX B}

\section{DERIVATION OF EQUATIONS (11) AND (12)}

To derive such equations we use the complex Gaussian moment theorem [31]:

Theorem 5: Let $U_{1}, U_{2}, \ldots, U_{2 k}$ be zero-mean jointly circular Gaussian random variables. Then

$$
\begin{aligned}
& E\left[U_{1}^{*} U_{2}^{*} \cdots U_{k}^{*} U_{k+1} U_{k+2} \cdots U_{2 k}\right] \\
& =\sum_{\pi} E\left[U_{\pi_{1}}^{*} U_{k+1}\right] E\left[U_{\pi_{2}}^{*} U_{k+2}\right] \cdots E\left[U_{\pi_{k}}^{*} U_{2 k}\right]
\end{aligned}
$$

where $\left(\pi_{1}, \pi_{2}, \ldots, \pi_{k}\right)$ are a permutation of $(1,2, \ldots, k)$ and $\sum_{\pi}$ indicates summation over all $k$ ! possible permutations.

In the special case of $k=3$ we have

$$
\begin{aligned}
& E\left[U_{1}^{*} U_{2}^{*} U_{3}^{*} U_{4} U_{5} U_{6}\right] \\
& \quad=E\left[U_{1}^{*} U_{4}\right]\left(E\left[U_{2}^{*} U_{5}\right] E\left[U_{3}^{*} U_{6}\right]+E\left[U_{2}^{*} U_{6}\right] E\left[U_{3}^{*} U_{5}\right]\right) \\
& \quad+E\left[U_{1}^{*} U_{5}\right]\left(E\left[U_{2}^{*} U_{4}\right] E\left[U_{3}^{*} U_{6}\right]+E\left[U_{2}^{*} U_{6}\right] E\left[U_{3}^{*} U_{4}\right]\right) \\
& \quad+E\left[U_{1}^{*} U_{6}\right]\left(E\left[U_{2}^{*} U_{4}\right] E\left[U_{3}^{*} U_{5}\right]+E\left[U_{2}^{*} U_{5}\right] E\left[U_{3}^{*} U_{4}\right]\right) .
\end{aligned}
$$

After substitution for $U_{1,4,5}=U(z, t+\tau)$ and $U_{2,3,6}=$ $U(s, t)$ we obtain four terms equal to $R_{z z}(0) R_{s s}(0) R_{z s}(\tau)$ and two terms equal to $\left|R_{z s}(\tau)\right|^{2} R_{z s}(\tau)$, with $R_{z s}(\tau)=$ $E\left[U(z, t+\tau) U^{*}(s, t)\right]$. The contributions sum up to (11).

In the eRP case, using $P(z)=2 R_{z z}(0)$ we have

$$
\begin{aligned}
R_{\mathrm{N}}(z, s, \tau)= & E\left[\mathcal{N}_{\mathrm{P}}(U(z, t+\tau)) \mathcal{N}_{\mathrm{P}}^{*}(U(s, t))\right] \\
= & E\left[\mathcal{N}_{0}(U(z, t+\tau)) \mathcal{N}_{0}^{*}(U(s, t))\right] \\
& -P(s) E\left[|U(z, t+\tau)|^{2} U(z, t+\tau) U^{*}(s, t)\right] \\
& -P(z) E\left[|U(s, t)|^{2} U^{*}(s, t) U(z, t+\tau)\right] \\
& +P(s) P(z) E\left[U(z, t+\tau) U^{*}(s, t)\right] .
\end{aligned}
$$

The first term on the right-hand side is (11). The last term is $4 R_{z z}(0) R_{s s}(0) R_{z s}(\tau)$. The two mid terms can be evaluated by the complex Gaussian moment theorem, obtaining:

$$
\begin{aligned}
E\left[|U(z, t+\tau)|^{2} U(z, t+\tau) U^{*}(s, t)\right] & =2 R_{z z}(0) R_{z s}(\tau) \\
E\left[|U(s, t)|^{2} U^{*}(s, t) U(z, t+\tau)\right] & =2 R_{s s}(0) R_{z s}(\tau)
\end{aligned}
$$

and hence (12) is obtained.

\section{APPENDIX C}

\section{EXTENSION TO DUAL POLARIZATION}

With two polarizations, we are dealing with a column vector electric field $\vec{A}(z, t)=\left[A_{x}(z, t), A_{y}(z, t)\right]$ propagating in an optical system that can be described by the Manakov equation. All linear operators can be described by matrices, which in absence of linear vectorial effects (e.g., PMD) remain proportional to the identity matrix. Each ACF generalizes into a correlation matrix. For instance, the key step (10) generalizes to evaluating the matrix:

$$
\begin{aligned}
\mathbf{R}_{\mathrm{N}}^{\mathrm{RP}}(z, s, \tau)= & E\left[\vec{A}^{\dagger}(z, t+\tau) \vec{A}(z, t+\tau) \vec{A}(z, t+\tau)\right. \\
& \left.\times \vec{A}^{\dagger}(s, t) \vec{A}^{\dagger}(s, t) \vec{A}(s, t)\right] .
\end{aligned}
$$

where $\dagger$ indicates transpose-conjugate. Introducing the matrix $\mathbf{R}_{z s}(\tau)=E\left[\vec{A}(z, t+\tau) \vec{A}^{\dagger}(s, t)\right]$ we can evaluate (35) by straightforward application of the complex Gaussian moment theorem in Appendix B, obtaining

$$
\mathbf{R}_{\mathrm{N}}^{\mathrm{RP}}(z, s, \tau)=\mathbf{P R}_{z s} \mathbf{P}^{\dagger}+\left(\operatorname{tr}\left[\mathbf{R}_{z s}^{\dagger} \mathbf{R}_{z s}\right] \mathbf{I}+\mathbf{R}_{z s} \mathbf{R}_{z s}^{\dagger}\right) \mathbf{R}_{z s}
$$

where $\mathbf{I}$ is the identity matrix, tr indicates trace of a matrix and $\mathbf{P} \triangleq \operatorname{tr}\left[\mathbf{R}_{z z}(0)\right] \mathbf{I}+\mathbf{R}_{z z}(0)$. Note that $\mathbf{P}$ does not depend on $z$ by the law of conservation of energy.

The nonlinear operator (5) in dual polarization becomes

$$
\mathcal{N}_{\mathrm{P}}(\vec{U})=\left(\vec{U}^{\dagger} \vec{U} \mathbf{I}-\hat{\mathbf{P}}\right) \vec{U}
$$

where $\hat{\mathbf{P}}$ is a positive semi-definite matrix that generalizes the scalar coefficient $\hat{P}$ of the scalar eRP to dual polarization. Generalizing the choice leading to (12), we choose $\hat{\mathbf{P}}$ in such a way as to remove any dependence on $\mathbf{R}_{z z}(0)$ and $\mathbf{R}_{s s}(0)$ in (36). This goal can be achieved by using $\hat{\mathbf{P}}=\mathbf{P}$. As a sanity check, note that $\mathbf{P}$ reduces to twice the average power in the scalar 
case, as in Fig. 1. In the eRP case we thus finally have

$$
\mathbf{R}_{\mathrm{N}}(z, s, \tau)=\left(\operatorname{tr}\left[\mathbf{R}_{z s}^{\dagger} \mathbf{R}_{z s}\right] \mathbf{I}+\mathbf{R}_{z s} \mathbf{R}_{z s}^{\dagger}\right) \mathbf{R}_{z s} .
$$

If $X$ and $Y$ polarizations are uncorrelated at $z=0$, when linear effects operate by a diagonal matrix, they remain uncorrelated at any $z$ since neither the nonlinear effect (37) nor the linear effect can create correlations. In this case, $\mathbf{R}_{z s}$ is a diagonal matrix, and thus it is exactly (37). For instance, the $(1,1)$ element of (37) becomes $\left(2 \mathbf{R}_{z s}^{(1,1)}+\mathbf{R}_{z s}^{(2,2)}\right) \mathbf{R}_{z s}^{(1,1)}$, where $\mathbf{R}_{z s}^{(i, j)}$ indicates element $(i, j)$ of matrix $\mathbf{R}_{z s}$. If $\mathbf{R}_{z s}$ is further proportional to I, i.e., $\mathrm{X}$ and $\mathrm{Y}$ are uncorrelated and carry the same PSD, we can still use the scalar theory introduced in Section III by using: i) a nonlinear index $\frac{8}{9} \gamma$ in place of $\gamma$; ii) a factor 3 in place of 2 in (12); iii) a per-polarization PSD $\tilde{G}_{0}(\omega)$, corresponding to $\tilde{G}_{\text {WDM }}(\omega) / 2$ of [10]. Overall, the PSD of the total NLI noise (adding X and Y PSDs) in dual uncorrelated polarizations is related to the total input PSD by replacing the factor 2 in (21) by $\left(\frac{8}{9}\right)^{2} \times 3 \times\left(\frac{1}{2}\right)^{3} \times 2=\frac{16}{27}[10]$. Note that in this case the ratio $\hat{\Phi} / \Phi_{\mathrm{NL}}$ of Fig. 1 saturates to $3 / 2$ instead of 2 .

\section{ACKNOWLEDGMENT}

The authors thank P. Johannisson for discussions on the GNRF and the connection with phase rotations of some of its terms, and L. Beygi for making them aware of the factor 2 in the average constellation phase rotation.

\section{REFERENCES}

[1] A. Carena, G. Bosco, V. Curri, P. Poggiolini, M. Tapia Taiba, and F. Forghieri, "Statistical Characterization of PM-QPSK Signals after Propagation in Uncompensated Fiber Links," presented at the Eur. Conf. Opt. Commun. 2010, Turin, Italy, Paper P4.07.

[2] F. Vacondio, O. Rival, C. Simonneau, E. Grellier, A. Bononi, L. Lorcy, J.-C. Antona, and S. Bigo, "On nonlinear distortions of highly dispersive optical coherent systems," Opt. Exp., vol. 2, no. 20, pp. 1022-1032, 2012.

[3] G. Bosco, A. Carena, R. Cigliutti, V. Curri, P. Poggiolini, and F. Forghieri, "Performance prediction for WDM PM-QPSK transmission over uncompensated links," presented at the Opt. Fiber Conf., 2011, Los Angeles, CA, USA, Paper OThO7.

[4] E. Grellier and A. Bononi, "Quality parameter for coherent transmissions with Gaussian-distributed nonlinear noise," Opt. Exp., vol. 19, no. 13, pp. 12781-12788, 2011.

[5] X. Chen and W. Shieh, "Closed-form expressions for nonlinear transmission performance of densely spaced coherent optical OFDM systems," Opt. Exp., vol. 18, pp. 19039-19054, 2010.

[6] A. Bononi, P. Serena, N. Rossi, E. Grellier, and F. Vacondio, "Modeling nonlinearity in coherent transmissions with dominant intrachannel-fourwave-mixing," Opt. Exp., vol. 20, no. 7, pp. 7777-7791, 2012.

[7] A. Mecozzi and R. J. Essiambre, "Nonlinear Shannon limit in pseudolinear coherent systems," J. Lightw. Technol., vol. 30, no. 12, pp. 2011-2024, 2012.

[8] A. Carena, V. Curri, G. Bosco, P. Poggiolini, and F. Forghieri, "Modeling of the impact of non-linear propagation effects in uncompensated optical coherent transmission links," J. Lightw. Technol., vol. 30, no. 10, pp. 15241539,2012

[9] L. Beygi, E. Agrell, P. Johannisson, M. Karlsson, H. Wymeersch, and P. Andrekson, "discrete-time model for uncompensated single-channel fiber-optical links," IEEE Trans. Commun., vol. 60, no. 11, pp. 34403450, Nov. 2012

[10] P. Poggiolini, "The GN model of non-linear propagation in uncompensated coherent optical systems," J. Lightw. Technol., vol. 30, no. 24, pp. 3857$3879,2012$.
[11] P. Serena and A. Bononi, "On the accuracy of the Gaussian nonlinear model for dispersion-unmanaged coherent links," presented at the Eur. Conf. Opt. Commun., 2013, London, U.K., Paper Th.1.D.3.

[12] P. Johannisson and M. Karlsson, "Perturbation analysis of nonlinear propagation in a strongly dispersive optical communication system," J. Lightw. Technol., vol. 31, no. 8, pp. 1273-1282, 2013.

[13] P. Poggiolini, G. Bosco, A. Carena, V. Curri, and F. Forghieri, (2012, Jul. 2), "A detailed analytical derivation of the GN model of non-linear interference in coherent optical transmission systems,"[Online]. Available: arXiv:1209.0394v2 [Phys. Opt.].

[14] A. Bononi and P. Serena, (2012, Jul. 19), "An alternative derivation of Johannisson's regular perturbation model,'[Online]. Available: arXiv:1207.4729v1 [Phys. Opt.]

[15] M. J. Ablowitz, T. Hirooka, and T. Inoue, "Higher-order asymptotic analysis of dispersion-managed transmission systems: Solutions and their characteristics," J. Opt. Soc. Amer. B, vol. 19, no. 12, pp. 2876-2885, 2002.

[16] A. Mecozzi, C. B. Clausen, and M. Shtaif, "Analysis of intrachannel nonlinear effects in highly dispersed optical pulse transmission," IEEE Photon. Technol. Lett., vol. 12, no. 4, pp. 392-394, Apr. 2000.

[17] P. M. Lushnikov, "Fully parallel algorithm for simulating dispersionmanaged wavelength-division-multiplexed optical fiber systems," Opt. Lett., vol. 27, no. 11, pp. 939-41, Jun. 2002.

[18] A. Vannucci, P. Serena, and A. Bononi, "The RP method: A new tool for the iterative solution of the nonlinear Schroedinger equation," J. Lightw. Technol., vol. 20, no. 7, pp. 1102-1112, 2002.

[19] M. Nazarathy, J. Khurgin, R. Weidenfeld, Y. Meiman, Pak Cho, R. Noe I. Shpantzer, and V. Karagodsky, "Phased-array cancellation of nonlinear FWM in coherent OFDM dispersive multi-span links," Opt. Exp., vol. 16, no. 20 , pp. $15778-15810,2008$.

[20] K. V. Peddanarappagari and M. Brandt-Pearce, "Volterra series transfer function of single-mode fibers," J. Lightw. Technol., vol. 15, no. 12, pp. 2232-2241, 1997.

[21] J. Tang, "The channel capacity of a multispan DWDM system employing dispersive nonlinear optical fibers and an ideal coherent optical receiver," J. Lightw. Technol., vol. 20, no. 7, pp. 1095-1101, 2002.

[22] C. Bender and S. Orszag, Advanced Mathematical Methods for Engineers. New York, NY, USA: McGraw-Hill, 1978.

[23] X. Wei, "Power-weighted dispersion distribution function for characterizing nonlinear properties of long-haul optical transmission links," Opt Lett., vol. 31, no. 17, pp. 2544-2546, 2006.

[24] G. P. Agrawal, Nonlinear Fiber Optics, 3rd ed. San Diego, CA, USA Academic, 2001

[25] E. Grellier, "Etude des effets non-linéaires à l'oeuvre dans les systèmes de transmissions optiques fortement dispersifs," Ph.D. dissertation, Dept. d'Optique, Université de Franche-comté, Besançon, France, 2011, p. 89.

[26] E. Ciaramella and E. Forestieri, "Analytical approximation of nonlinear distortions," IEEE Photon. Technol. Lett., vol. 17, pp. 91-93, Jan. 2005.

[27] B. Xu and M. Brandt-Pearce, "Modified Volterra series transfer function method," IEEE Photon. Technol.Lett., vol. 14, no. 1, pp. 47-49, Jan. 2002.

[28] H. Louchet, A. Hodzic, K. Petermann, A. Robinson, and R. Epworth, "Simple criterion for the characterization of nonlinear impairments in dispersion-managed optical transmission systems," IEEE Photon. Technol. Lett., vol. 17, no. 10, pp. 2089-2091, Oct. 2005.

[29] A. O. Korotkevich and P. M. Lushnikov, "Proof-of-concept implementation of the massively parallel algorithm for simulation of dispersionmanaged WDM optical fiber systems.," Opt. Lett., vol. 36, no. 10 pp. 1851-1853, May 2011.

[30] A. Papoulis, Probability, Random Variables, and Stochastic Processes, 3rd ed. New York, NY, USA: McGraw-Hill, 1991.

[31] I. S. Reed, "On a Moment Theorem for Complex Gaussian Processes," IRE Trans. Inform. Theory, vol. 8, no. 3, pp. 194-195, Apr. 1962.

[32] A. Bononi, N. Rossi, and P. Serena, "On the nonlinear threshold versus distance in long-haul highly-dispersive coherent systems," Opt. Exp., vol. 20, no. 26, pp. B204-B216, 2012.

[33] S. J. Savory, "Digital filters for coherent optical receivers.," Opt. Exp., vol. 16, no. 2, pp. 804-817, Jan. 2008.

[34] R. Weinstock, Calculus of Variations With Applications to Physics and Engineering. New York, NY, USA: Dover, 1974.

Authors' photographs and biographies not available at the time of publication. 